\title{
OSCILLATION THEOREMS FOR $n$ TH ORDER NONLINEAR DIFFERENTIAL EQUATIONS WITH DEVIATING ARGUMENTS
}

\author{
S. R. GRACE AND B. S. LALLI
}

\begin{abstract}
In this note we study the oscillatory behavior of solutions of the $n$th order nonlinear functional differential equation

$$
x^{(n)}(t)+q(t) f(x[g(t)])=0, \quad n \text { even, }
$$

without assuming that the deviating argument is retarded or advanced. Sufficient conditions are established for all solutions of the equation to be oscillatory.
\end{abstract}

The purpose of this note is to obtain some oscillation criteria for the $n$th order equation

$$
x^{(n)}(t)+q(t) f(x[g(t)])=0, \quad n \text { even, }
$$

where the following conditions are assumed to hold:

(2) $q, g \in C\left[\left[t_{0}, \infty\right), R\right], q(t)$ is nonnegative and not identically zero on any interval of the form $\left[t_{1}, \infty\right), t_{1} \geqslant t_{0}$ and $\lim _{t \rightarrow \infty} g(t)=\infty$;

(3) $f \in C[R, R], f$ is nondecreasing $0<f(x) \leqslant-f(-x)$ for $x>0$;

(4) $\int_{0}^{\alpha} d u / f(u)<\infty$ and $\int_{-0}^{-\alpha} d u / f(u)<\infty$ for any $\alpha>0$;

(5) $f(x y) \geqslant K f(x) f(y)$ for $x, y>0$ and $K$ is a positive constant.

In what follows, by a proper solution of (1), we mean a function $x \in$ $C^{n}\left[\left[T_{x}, \infty\right), R\right]$ which satisfies (1) for all sufficiently large $t$ and $\sup \{|x(t)|: t \geqslant T\}$ $>0$ for any $T \geqslant T_{x}$. A proper solution of (1) is called oscillatory if it has arbitrarily large zeros and it is called nonoscillatory otherwise.

We will have an occasion to use the following two lemmas given in [1].

LEMMA 1. Let $u$ be a positive and n-times differentiable function on an interval $\left[t_{0}, \infty\right)$. If $u^{(n)}$ is of constant sign and not identically zero on any interval of the form $\left[t_{1}, \infty\right), t_{1} \geqslant t_{0}$, then there exists a $t_{u} \geqslant t_{0}$ and an integer $l, 0 \leqslant l \leqslant n$ with $n+l$ even for $u^{(n)} \geqslant 0$ or $n+l$ odd for $u^{(n)} \leqslant 0$ and such that $l>0$ implies $u^{(k)}(t)>0$ for every $t \geqslant t_{u}(k=0,1, \ldots, l-1)$ and $l \leqslant n-1$ implies $(-1)^{l+k} u^{(k)}(t)>0$ for every $t \geqslant t_{u}$ $(k=l, l+1, \ldots, n-1)$.

LEMMA 2. If the function $u$ is as in Lemma 1 and

$$
u^{(n-1)}(t) u^{(n)}(t) \leqslant 0 \text { for every } t \leqslant t_{u},
$$

Received by the editors January 18, 1982 and, in revised form, September 8, 1982

1980 Mathematics Subject Classification. Primary 34K25. 
then for every $\lambda, 0<\lambda<1$, there exists an $M_{\lambda}>0$ such that

$$
u[\lambda t] \geqslant M_{\lambda} t^{n-1}\left|u^{(n-1)}(t)\right| \text { for all large } t .
$$

Moreover, if $u$ is increasing, then

$$
u(t) \geqslant u\left[\frac{1}{2} t\right] \geqslant M_{1 / 2} t^{n-1}\left|u^{(n-1)}(t)\right| \text { for all large } t .
$$

REMARK. From the proof of Lemma 2 [1, Lemma 2], we can take

$$
M_{1 / 2}=\frac{2^{2-2^{n}}}{(n-1) !} \text {. }
$$

We also need the following lemma.

Lemma 3. Let $n=1$, and conditions (2)-(4) hold. If

$$
\int_{A} q(s) d s=\infty
$$

where $A=\left\{t \in\left[t_{0}, \infty\right): t_{0} \leqslant g(t)<t\right\}$, the retarded part of $g(t)$, then all proper solutions of (1) are oscillatory.

Proof. The proof is similar to that of Theorem 2 in [3] and hence is omitted.

THEOREM 1. Let conditions (2)-(5) hold. Then all proper solutions of (1) are oscillatory if

$$
\int_{A} q(s) f\left(g^{n-1}(s)\right) d s=\infty,
$$

where $A=\left\{t \in\left[t_{0}, \infty\right): t_{0} \leqslant g(t)<t\right\}$, the retarded part of $g(t)$.

Proof. Let $x(t)$ be a nonoscillatory solution of (1), say $x(t)>0$ for $t \geqslant t_{1} \geqslant t_{0}$. Then there exists $t_{2} \geqslant t_{1}$ such that $x[g(t)]>0$ for $t \geqslant t_{2}$.

By Lemma 1 , there exists a $t_{3} \geqslant t_{2}$ such that

$$
\dot{x}(t)>0 \text { and } x^{(n-1)}(t)>0 \text { for } t \geqslant t_{3} .
$$

Next, applying Lemma 2 for $u=x$, we derive that there exists a $t_{4} \geqslant t_{3}$ such that

$$
x(t) \geqslant M_{1 / 2} t^{n-1} x^{(n-1)}(t) \text { for } t \geqslant t_{4} .
$$

Consequently, since $g(t) \rightarrow \infty$ as $t \rightarrow \infty$, there exists a $t_{5} \geqslant t_{4}$ such that

$$
x[g(t)] \geqslant M_{1 / 2} g^{n-1}(t) x^{(n-1)}[g(t)] \text { for } t \geqslant t_{5} .
$$

Thus (1) becomes

$$
x^{(n)}(t)+q(t) f\left(M_{1 / 2} g^{n-1}(t) x^{(n-1)}[g(t)]\right) \leqslant 0 \text { for } t \geqslant t_{5} .
$$

Now, using (5) we get

$$
x^{(n)}(t)+K^{2} q(t) f\left(M_{1 / 2}\right) f\left(g^{n-1}(t)\right) f\left(x^{(n-1)}[g(t)]\right) \leqslant 0
$$

or

$$
x^{(n)}(t)+c q(t) f\left(g^{n-1}(t)\right) f\left(x^{(n-1)}[g(t)]\right) \leqslant 0
$$


for some constant $c>0$. Now if we let $u(t)=x^{(n-1)}(t)$, then (9) reduces to

$$
\dot{u}(t)+c q(t) f\left(g^{n-1}(t)\right) f(u[g(t)]) \leqslant 0 .
$$

Using Lemma 3, we obtain the desired contradiction.

If $x(t)<0$ for $t \geqslant t_{1} \geqslant t_{0}$, then the transformation $y=-x$ transforms (1) to

$$
y^{(n)}(t)+q(t) f^{*}(y[g(t)])=0, \quad n \text { even, }
$$

where $f^{*}(y)=-f(-y)$. Thus the above argument can be repeated on the positive solutions $y(t)$ of $\left(1^{\prime}\right)$; this in turn yields the required results for negative solutions of (1).

For illustration consider the following example.

EXAmple 1. Consider the equation

$$
x^{(6)}(t)+(t-1)^{-8 / 3} x^{1 / 3}[t+\sin t]=0, \quad t \geqslant \pi .
$$

The retarded part of $g(t)=t+\sin t$ is $A=\cup_{k=1}^{\infty}((2 k-1) \pi, 2 k \pi)$.

$$
\begin{aligned}
\int_{A} q(s) f\left(g^{n-1}(s)\right) d s & =\sum_{k=1}^{\infty} \int_{(2 k-1) \pi}^{2 k \pi} \frac{(t+\sin t)^{5 / 3}}{(t-1)^{8 / 3}} d t \\
& \geqslant \sum_{k=1}^{\infty} \int_{(2 k-1) \pi}^{2 k \pi} \frac{(t-1)^{5 / 3}}{(t-1)^{8 / 3}} d t \\
& =\sum_{k=1}^{\infty} \log \frac{2 k \pi-1}{2 k \pi-\pi-1}=\infty,
\end{aligned}
$$

and so from Theorem 1, it follows that all proper solutions of (1) are oscillatory.

The following theorem extends and improves Theorem 3.2 of Wong [9] who considered the equation

$$
\ddot{x}(t)+q(t) x[g(t)]=0,
$$

where $c t \leqslant g(t) \leqslant t, c>0$ and $q(t) \geqslant 0$.

THEOREM 2. Let conditions (2) and (3) hold, and

$$
f^{\prime}(x)>\alpha>0 \text { for } x \neq 0\left({ }^{\prime}=d / d t\right) .
$$

Suppose further that there exist functions $\rho, \sigma \in C^{1}\left[\left[t_{0}, \infty\right),(0, \infty)\right]$ such that

$$
\sigma(t) \leqslant \min \{t, 2 g(t)\}, \dot{\sigma}(t)>0 \text { and } \sigma(t) \rightarrow \infty \text { as } t \rightarrow \infty(\cdot=d / d t)
$$

and

$$
\lim _{t \rightarrow \infty} \sup \int_{t_{0}}^{t}\left[\rho(s) q(s)-\frac{\dot{\rho}^{2}(s)}{2 \alpha M_{1 / 2} \sigma^{n-2}(s) \dot{\sigma}(s) \rho(s)}\right] d s=\infty,
$$

where $M_{1 / 2}=2^{2-2 n} /(n-1) !\left(M_{1 / 2}\right.$ is as in Lemma 2 for $\left.\lambda=\frac{1}{2}\right)$. Then every solution of equation (1) is oscillatory.

Proof. Let $x(t)$ be a nonoscillatory solution of (1), say $x(t)>0$ for $t \geqslant t_{1} \geqslant t_{0}$. It follows, as in the proof of Theorem 1 that there exists a $t_{3} \geqslant t_{2}$ so that

$$
\dot{x}(t)>0 \text { and } x^{(n-1)}(t)>0 \text { for } t \geqslant t_{3} \text {. }
$$


Now, applying Lemma 2 for $u=\dot{x}$ and $\lambda=\frac{1}{2}$, we easily derive that there exists $t_{4} \geqslant t_{3}$ such that

$$
\dot{x}\left[\frac{1}{2} t\right] \geqslant M_{1 / 2} t^{n-2} x^{(n-1)}(t) \text { for } t \geqslant t_{4} .
$$

Since $\sigma(t) \rightarrow \infty$ as $t \rightarrow \infty$, there exists $t_{5} \geqslant t_{4}$ such that

$$
\dot{x}\left[\frac{1}{2} \sigma(t)\right] \geqslant M_{1 / 2} \sigma^{n-2}(t) x^{(n-1)}[\sigma(t)] \text { for } t \geqslant t_{5} .
$$

Now, by condition (13) and the fact that $x^{(n-1)}(t)$ is nonincreasing, we have

$$
\dot{x}\left[\frac{1}{2} \sigma(t)\right] \geqslant M_{1 / 2} \sigma^{n-2}(t) x^{(n-1)}(t) \text { for } t \geqslant t_{5} .
$$

Let

$$
w(t)=\frac{\rho(t) x^{(n-1)}(t)}{f\left(x\left[\frac{1}{2} \sigma(t)\right]\right)}
$$

Thus $w(t)$ satisfies

$$
\begin{aligned}
\dot{w}(t) \leqslant & -\rho(t) q(t) \frac{f(x[g(t)])}{f(x[\sigma(t) / 2])}+\frac{\dot{\rho}(t)}{\rho(t)} w(t) \\
& -\frac{1}{2} f^{\prime}\left(x\left[\frac{1}{2} \sigma(t)\right]\right) w(t) \frac{\dot{x}[\sigma(t) / 2] \dot{\sigma}(t)}{f(x[\sigma(t) / 2])} \\
\leqslant & -\rho(t) q(t)+\frac{\dot{\rho}(t)}{\rho(t)} w(t)-\frac{1}{2} M_{1 / 2} \alpha \frac{\sigma^{n-2}(t) \dot{\sigma}(t)}{\rho(t)} w^{2}(t) \\
\leqslant & -\rho(t) q(t)+\frac{\dot{\rho}^{2}(t)}{2 M_{1 / 2} \alpha \sigma^{n-2}(t) \dot{\sigma}(t) \rho(t)} \\
& -\left(\left(\frac{1}{2} M_{1 / 2} \alpha \frac{\sigma^{n-2}(t) \dot{\sigma}(t)}{\rho(t)}\right)^{1 / 2} w(t)\right. \\
& \left.-\frac{\dot{\rho}(t) / 2 / \rho(t)}{\left(\left(M_{1 / 2} \alpha / 2\right) \sigma^{n-2}(t) \dot{\sigma}(t) / \rho(t)\right)^{1 / 2}}\right)^{2} \\
\leqslant & -\rho(t) q(t)+\frac{\dot{\rho}^{2}(t)}{2 M_{1 / 2} \alpha \sigma^{n-2}(t) \dot{\sigma}(t) \rho(t)} .
\end{aligned}
$$

Integrate the above inequality from $t_{5}$ to $t$ to obtain

$$
\int_{t_{5}}^{t}\left[\rho(s) q(s)-\frac{\dot{\rho}^{2}(s)}{2 M_{1 / 2} \alpha \sigma^{n-2}(s) \dot{\sigma}(s) \rho(s)}\right] d s \leqslant w\left(t_{5}\right)-w(t) \leqslant w\left(t_{5}\right)<\infty
$$

for all $t \leqslant t_{5}$. This contradicts (14). The case $x(t)<0$ for $t \geqslant t_{1} \geqslant t_{0}$ is similar and hence is omitted.

COROLlaRY. Let the condition (14) in Theorem 2 be replaced by

$$
\lim _{t \rightarrow \infty} \sup \int_{t_{0}}^{t} \rho(s) q(s) d s=\infty
$$


and

$$
\lim _{t \rightarrow \infty} \int_{t_{0}}^{t} \frac{\dot{\rho}^{2}(s)}{\rho(s) \sigma^{n-2}(s) \dot{\sigma}(s)} d s<\infty
$$

Then the conclusion of Theorem 2 holds.

In the following theorem the function $f$ is not required to be differentiable.

THEOREM 3. Let the conditions (3), (12) and (14) in Theorem 2 be replaced by

$$
f \in C[R, R], x f(x)>0 \text { and } f(x) / x \geqslant c_{1}>0 \text { for } x \neq 0
$$

and

$$
\lim _{t \rightarrow \infty} \sup \int_{t_{0}}^{t}\left[c_{1} \rho(s) q(s)-\frac{\dot{\rho}^{2}(s)}{2 M_{1 / 2} \rho(s) \sigma^{n-2}(s) \dot{\boldsymbol{\sigma}}(s)}\right] d s=\infty,
$$

respectively, then the conclusion of Theorem 2 holds.

Proof. Let $x(t)$ be a nonoscillatory solution of (1). Assume $x(t)>0$ for $t \geqslant t_{1} \geqslant$ $t_{0}$. As in the proof of Theorem 2 we have

$$
\dot{x}(t)>0 \text { and } x^{(n-1)}(t)>0 \text { for } t \geqslant t_{3},
$$

and

$$
\dot{x}[\sigma(t) / 2] \geqslant M_{1 / 2} \sigma^{n-2}(t) x^{(n-1)}(t) \text { for } t \geqslant t_{5} .
$$

Letting

$$
w(t)=\frac{\rho(t) x^{(n-1)}(t)}{x[\sigma(t) / 2]}
$$

we get

$$
\dot{w}(t) \leqslant-c_{1} \rho(t) q(t)+\frac{\dot{\rho}(t)}{\rho(t)} w(t)-\frac{1}{2} M_{1 / 2} \frac{\sigma^{n-2}(t) \dot{\sigma}(t)}{\rho(t)} w^{2}(t) .
$$

The rest of the proof is similar to that of Theorem 2 and hence is omitted.

The following example is illustrative.

EXAMPLE 2. Consider the equations

$$
x^{(n)}(t)+\gamma t^{-n} x[c t]=0 \text { for } n \text { even, } t>0
$$

and

$$
\begin{aligned}
& x^{(n)}(t)+\gamma t^{-n} x[c t] \exp (\sin x[c t])=0 \text { for } n \text { even, } t>0, \\
& \text { where } c \text { and } \gamma \text { are positive constants. We let }
\end{aligned}
$$

$$
\sigma(t)= \begin{cases}c t, & 0<c<1 \\ t, & c \geqslant 1\end{cases}
$$

Equation (20) is oscillatory by Theorem 2 for $\rho(t)=t^{n-1}$ and

$$
\gamma>c^{1-n} 2^{2 n-3}(n-1) !(n-1)^{2}
$$


and equation (21) is oscillatory by Theorem 3 for $\rho(t)=t^{n-1}, c_{1}=e^{-1}$ and

$$
\gamma>e c^{1-n} 2^{2 n-3}(n-1) !(n-1)^{2} .
$$

It can easily be verified that Theorem 2.5(ii) in [2] and Theorem 1(ii) in [8] are not applicable to equation (20).

RemarKs. 1. Theorem 1 improves some of the results in $[\mathbf{2}, \mathbf{4}, \mathbf{5}, \mathbf{7}$ and $\mathbf{8}]$ for the case when $f(x)=x^{\alpha} \operatorname{sgn} x, 0<\alpha<1$, while Theorems 2 and 3 unify and improve Theorem 1(ii) in [8] and Theorem 2.5(ii) in [2].

2. It is easy to check that our Theorems 2 and 3, when specialized to equation (11), turn out to be a substantial improvement on Theorem 3.2 in [9] and a result in [6].

3. It is clear that we do not stipulate that the function $g$ in equation (1) be either retarded or advanced. Hence our theorems may hold for ordinary, retarded, advanced, and mixed type equations (see Examples 1 and 2 above).

4. The results of this paper can be extended to equations of the form

$$
x^{(n)}(t)+\sum_{i=1}^{m} q_{i}(t) f_{i}\left(x\left[g_{i}(t)\right]\right)+F\left(t, x(t), x\left[g_{1}(t)\right], \ldots, x\left[g_{m}(t)\right]\right)=0,
$$

$n$ is even, where $q_{i}, g_{i} \in C\left[\left[t_{0}, \infty\right), R\right], f_{i} \in C[R, R], q_{i}(t), g_{i}(t)$ and $f_{i}(x), 1 \leqslant i \leqslant m$, satisfy the same assumptions we impose on $q(t), g(t)$ and $f(x)$ respectively, and $F \in C\left[\left[t_{0}, \infty\right) \times R^{m+1}, R\right]$ satisfies

$$
y_{0} F\left(t, y_{0}, y_{1}, \ldots, y_{m}\right) \geqslant 0 \text { for } y_{0} y_{i}>0,1 \leqslant i \leqslant m .
$$

ACKNOWLEDGEMENT. The authors are indebted to the referee for a number of helpful comments and suggestions.

\section{REFERENCES}

1. M. K. Grammatikopoulos, Y. G. Sficas and V. A. Staikos, Oscillatory properties of strongly. superlinear differential equations with deviating arguments, J. Math. Anal. Appl. 67 (1979), 171- 187.

2. A. G. Kartsatos, Recent results on oscillation of solutions of forced and perturbed nonlinear differential equations of even order, Stability of Dynamical Systems: Theory and Applications, Lecture Notes in Pure and Appl. Math., vol. 28, Dekker, New York, 1977, pp. 17-72.

3. Y. Kitamura and T. Kusano, Oscillation of first order nonlinear differential equations with deviating arguments, Proc. Amer. Math. Soc. 78 (1980), 64-68.

4. T. Kusano and $\mathrm{H}$. Onose, Oscillaton and asymptotic hehavior of sublinear retarded differential equations, Hiroshima Math. J. 4 (1974), 343-355.

5. I. Ličko and M. Švec, Le caractere oscillatoire des solutions de l'equation $y^{(n)}+f(x) y^{\alpha}=0, n>1$, Czechoslovak Math. J. 13 (1963), 481-489.

6. Z. Opial, Sur une critére d'oscillation différentielle $\left(Q(t) x^{\prime}\right)^{\prime}+f(t) x=0$, Ann. Polon. Math. 6 (1959), 99-104.

7. Y. G. Sficas and V. A. Staikos, Oscillations of retarded differential equations, Math. Proc. Cambridge Philos. Soc. 75 (1974), 95-101.

8. U. Sztaba, Note on the Dahiya and Singh's paper: on oscillation behavior of even order delay equations, J. Math. Anal. Appl. 63 (1978), 313-318.

9. J. S. W. Wong, Second order oscillation with retarded arguments, Ordinary Differential Equations, Academic Press, New York, 1972, pp. 581-596.

Department of Mathematics, University of Saskatchewan, Saskatoon, Saskatchewan S7N OW0, CANADA (Current address of B. S. Lalli)

Current address (S. R. Grace): Department of Mathematics, Faculty of Engineering, Cairo University, Orman, Giza, Egypt 